SUPPORTING INFORMATION

August 8, 2020

\title{
Magnetic Coupling in a Tris-hydroxo-bridged Chromium Dimer Occurs Through Ligand Mediated Superexchange in Conjunction with Through- Space Coupling
}

\author{
Prachi Sharma, Donald G. Truhlar, * Laura Gagliardi* \\ Department of Chemistry, Chemical Theory Center, and Minnesota Supercomputing Institute, \\ 207 Pleasant Street SE, University of Minnesota, Minneapolis, MN 55455-0431 \\ * Corresponding authors
}

TABLE OF CONTENTS

Figure S1. Natural molecular orbitals obtained from CASSCF $(6,10) S=0$ calculation

Figure S2. Natural molecular orbitals obtained from CASSCF $(6,10) S=1$ calculation

Figure S3. Natural molecular orbitals obtained from CASSCF $(6,10) S=2$ calculation

Figure S4. Natural molecular orbitals obtained from RASSCF $(30,22) S=3$ calculation

Figure S5. Natural molecular orbitals obtained from CASSCF $(30,22) S=0$ calculation

Figure S6. Natural molecular orbitals obtained from CASSCF $(30,22) S=1$ calculation

Figure S7. Natural molecular orbitals obtained from CASSCF $(30,22) S=2$ calculation

Figure S8. Natural molecular orbitals obtained from CASSCF $(30,22) S=3$ calculation

Table S1. Occupation numbers of CASSCF $(6,10)$ orbitals

Table S2. Occupation numbers of RASSCF $(30,22)$ orbitals

Table S3. Occupation numbers of DMRG-SCF $(30,22)$ orbitals 
Figure S9. Comparison between ligand orbitals in CASSCF vs RASSCF

Table S4. Absolute MCSCF and MC-PDFT energies in atomic units

Table S5. Percentage contribution of $\mathrm{Cr}(3 \mathrm{~d})$ and $\mathrm{O}$ atomic orbitals to selected DMRG molecular orbitals

Table S6 Relative energies of spin states and coupling constant $(J)$ in $\mathrm{cm}^{-1}$ for the modified chromium dimer without the bridging hydroxide ligands

Table S7 Comparison between occupation numbers of Kremer's dimer and dimer with deleted hydroxide ligands.

Figure S10. Unpaired density for CAS-PDFT (left) and DMRG1-PDFT (right) for Kremer's complex in (a) the $Y Z$-plane, (b) the $X Z$-plane, and (c) the $X Y$-plane.

Figure S11. Unpaired density for CAS-PDFT (left) and RAS-PDFT (right) for Kremer's complex in (a) the $Y Z$-plane, (b) the $X Z$-plane, and (c) the $X Y$-plane.

Figure S10. Unpair electron density difference between $S=3$ (RAS-PDFT) and S=0 (RASPDFT)

Note: Input and RasOrb files, which are editable files produced by the RASSCF program of OpenMolcas to allow the possibility to read the orbitals in a later run, are available as separate TXT files. 


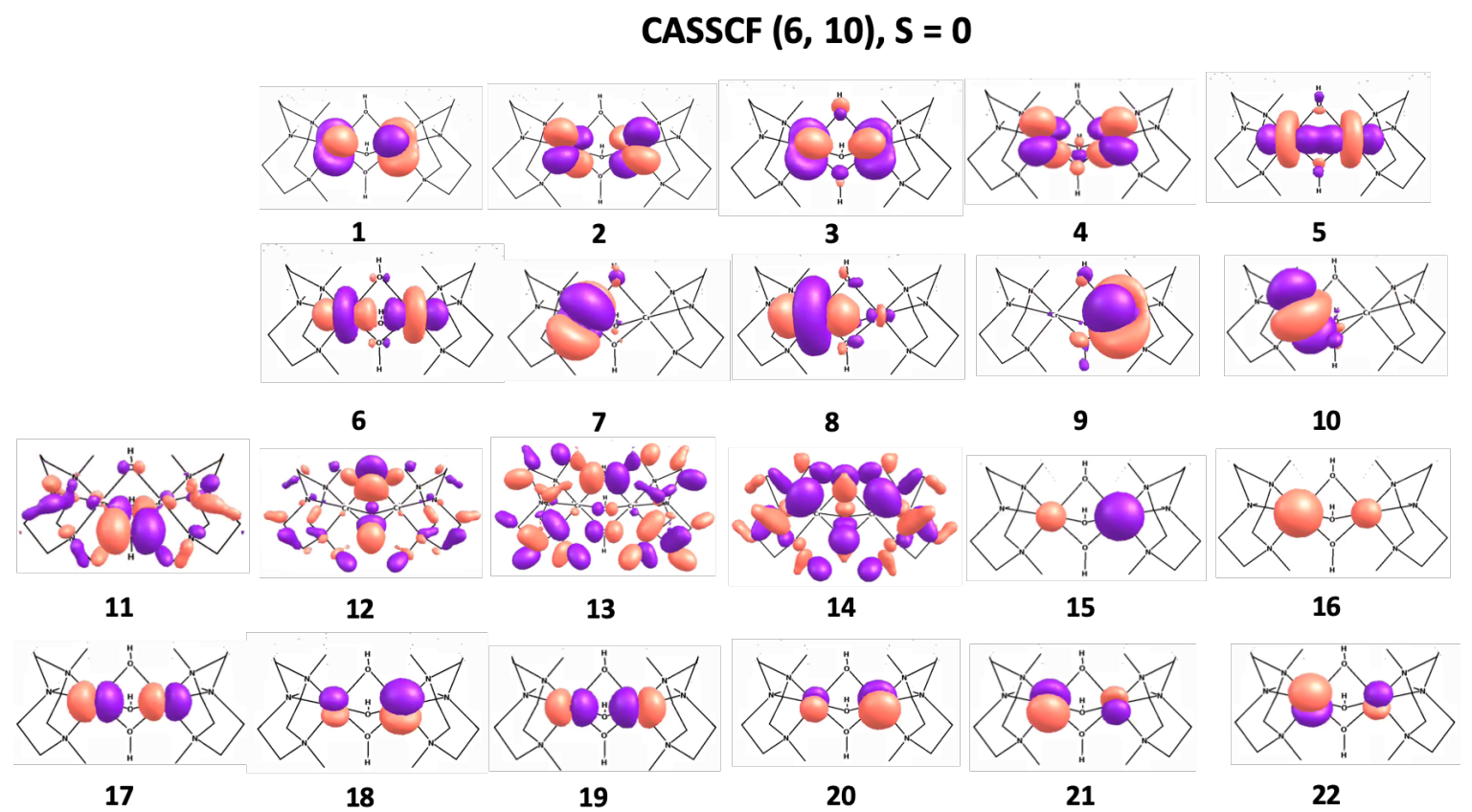

Figure S1. Natural molecular orbitals obtained from CASSCF $(6,10), S=0$ calculation. Orbitals 1-10 represents active space while 11-22 are inactive orbitals .

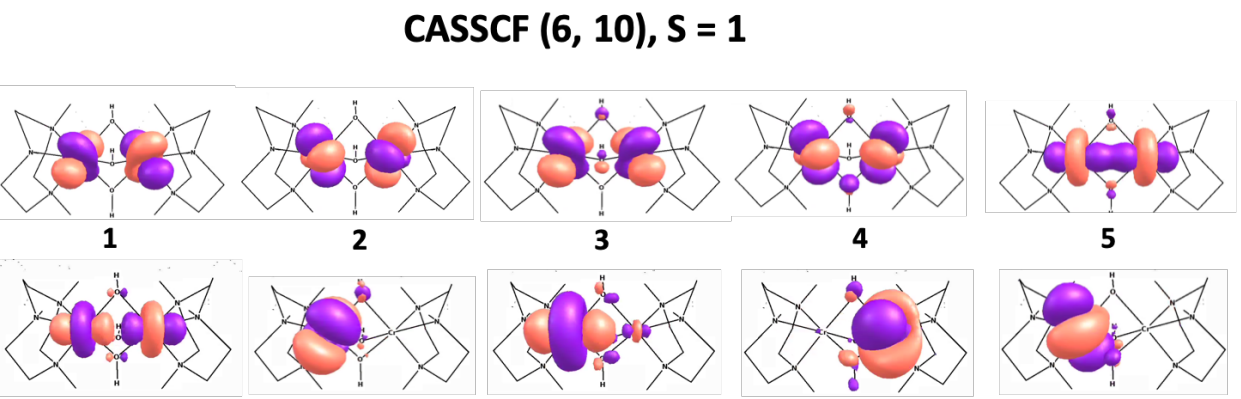

6

$\begin{array}{ll}7 & 8\end{array}$

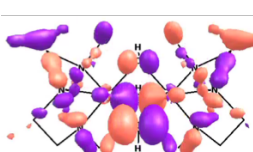

11

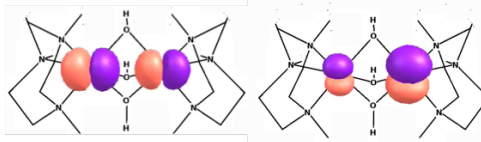

17
12

13

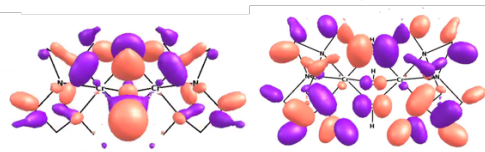

13

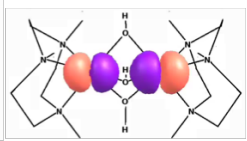

19
18

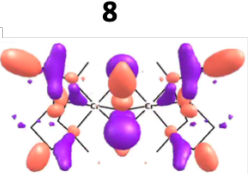

14

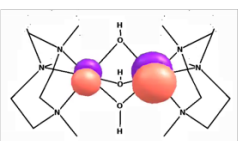

20
9

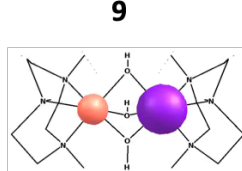

15

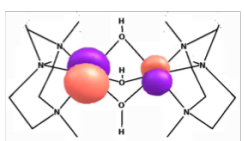

21
10

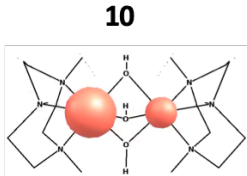

16

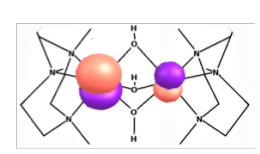

22

Figure S2. Natural molecular orbitals obtained from CASSCF $(6,10), S=1$ calculation. Orbitals 1-10 represents active space while 11-22 are inactive orbitals . 


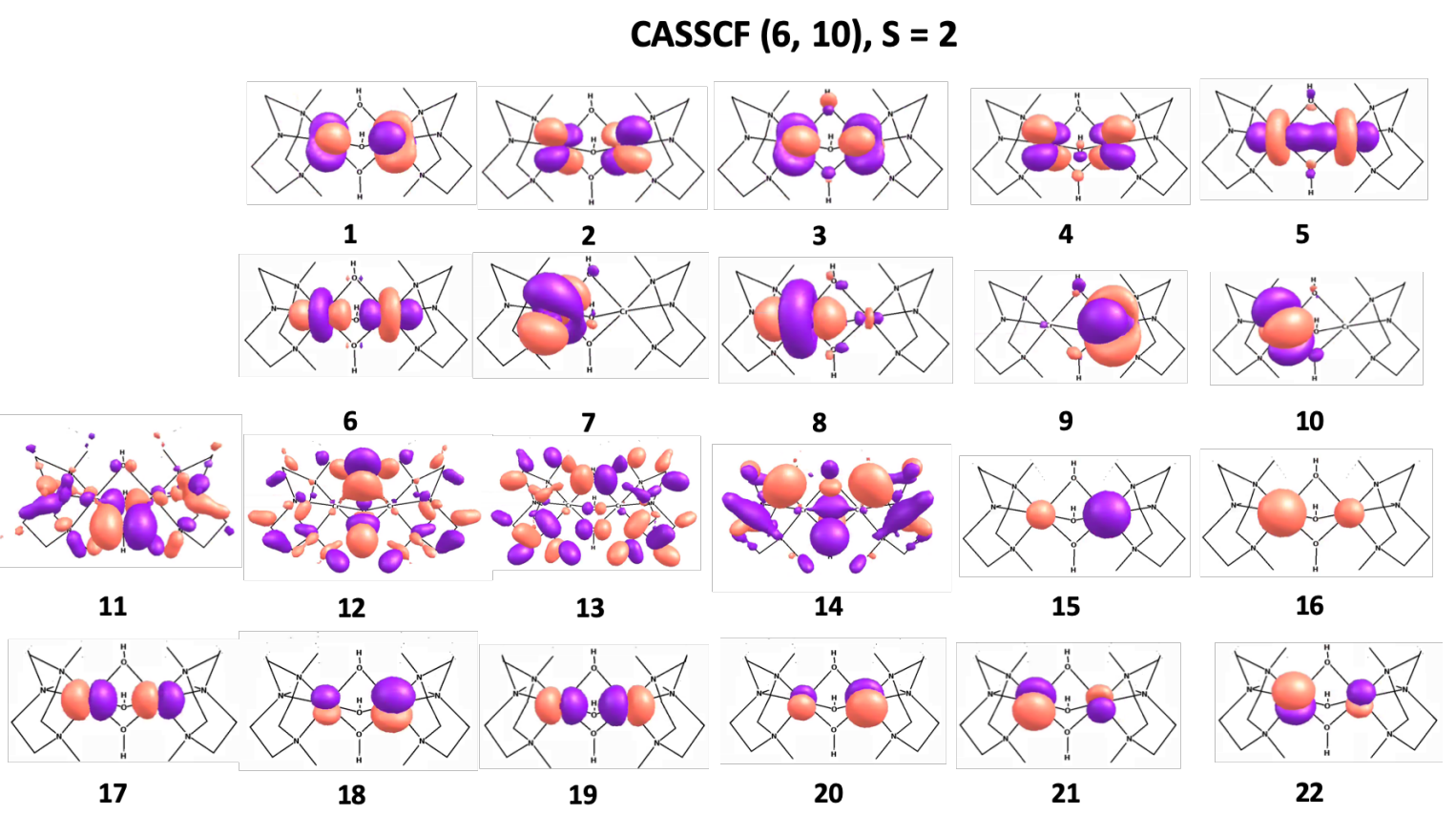

Figure S3. Natural molecular orbitals obtained from CASSCF $(6,10), S=2$ calculation. Orbitals 1-10 represents active space while 11-22 are inactive orbitals .

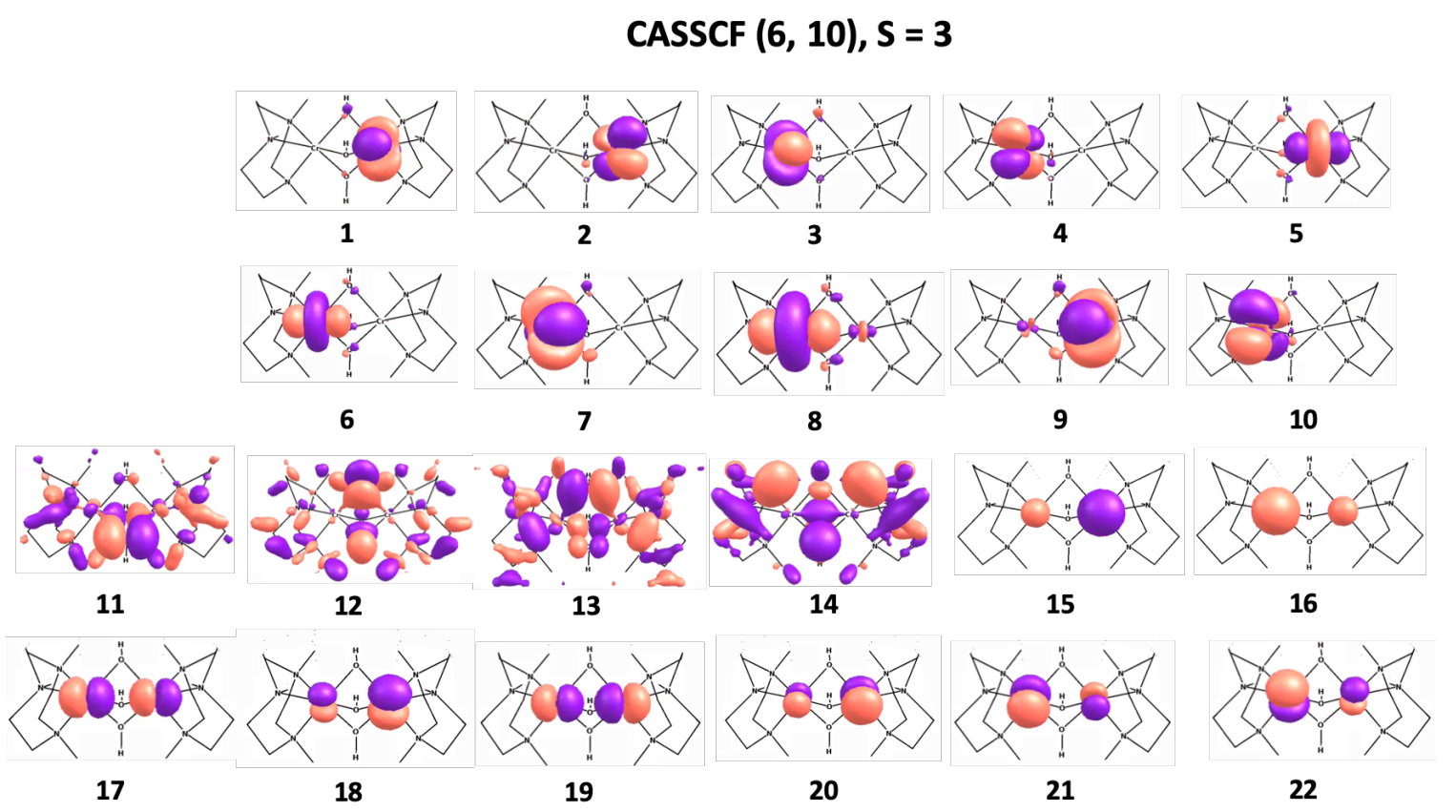

Figure S4. Natural molecular orbitals obtained from CASSCF $(6,10), \mathrm{S}=3$ calculation. Orbitals 1-10 represents active space while 11-22 are inactive orbitals . 
$\operatorname{RASSCF}(30,22), S=0$

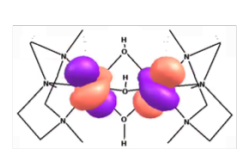

1

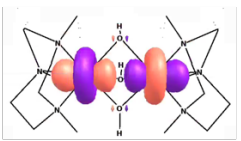

6

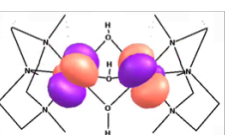

2

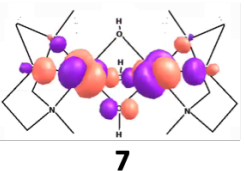

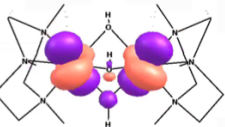

3

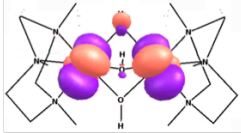

4

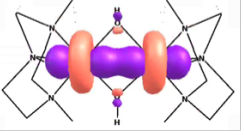

5 nerenter

11

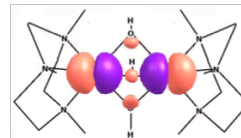

17

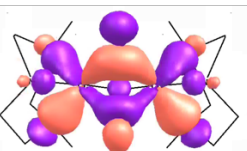

12

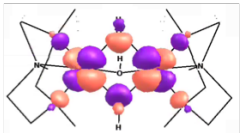

8
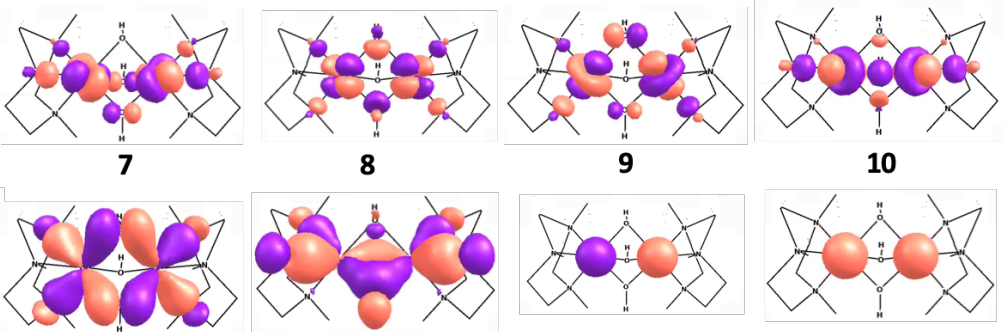

13
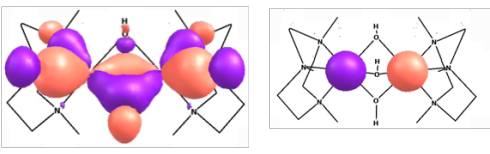

15

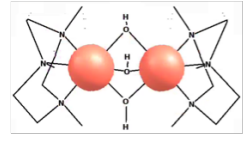

16

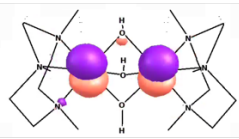

18

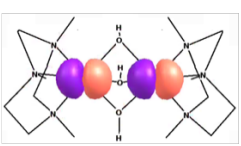

19

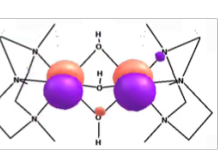

20

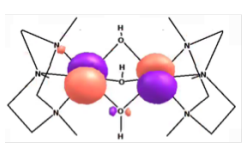

21

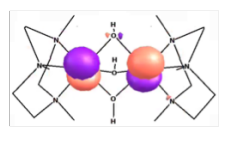

22

Figure S5. Natural molecular orbitals obtained from RASSCF $(30,22), S=0$ calculation.

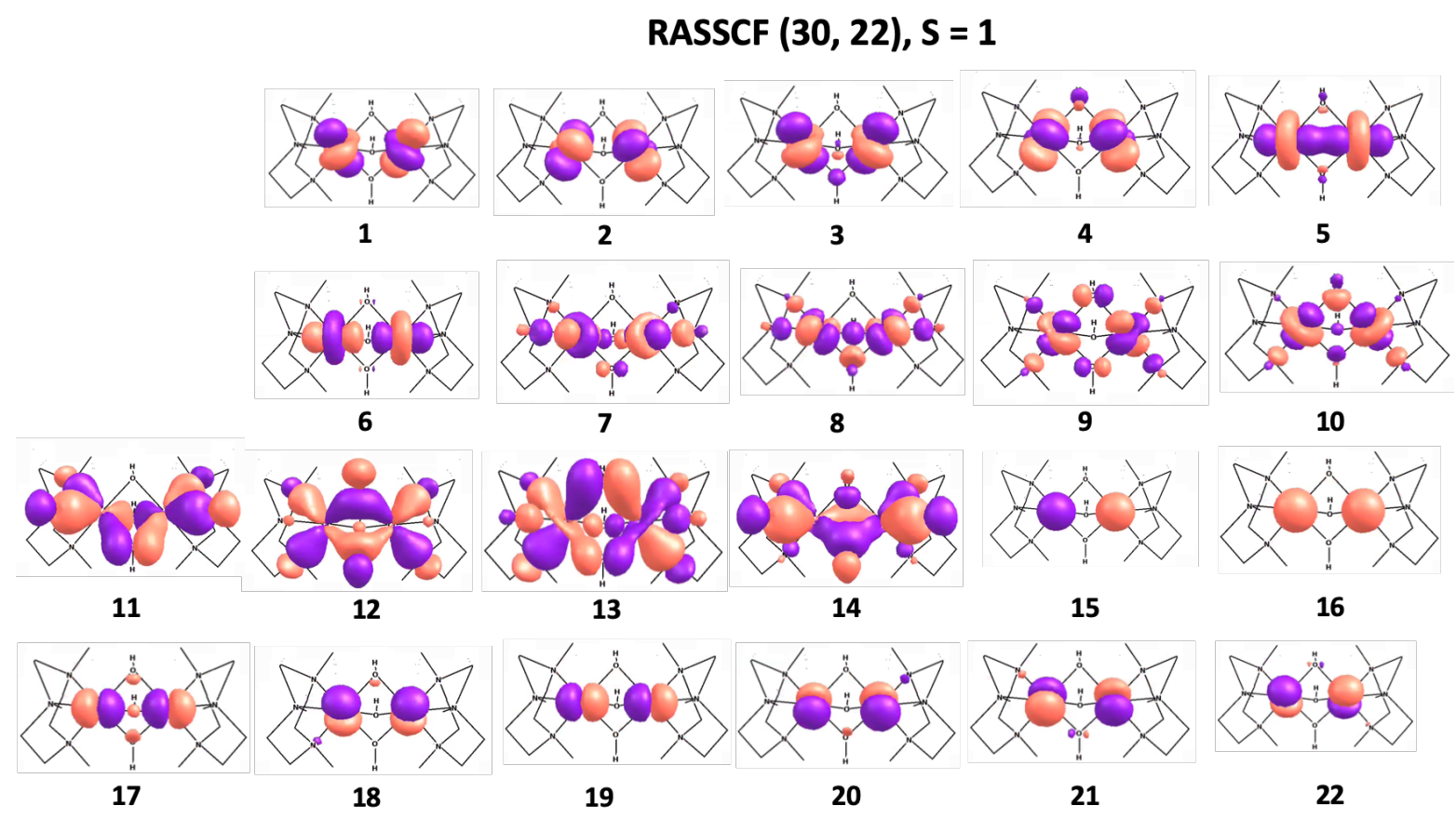

Figure S6. Natural molecular orbitals obtained from RASSCF (30, 22), $S=1$ calculation. 
$\operatorname{RASSCF}(30,22), S=2$

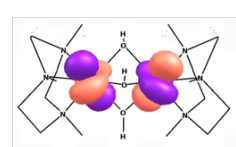

1

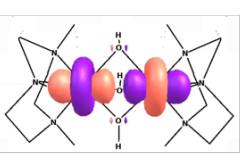

6

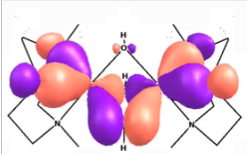

11

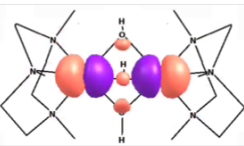

17

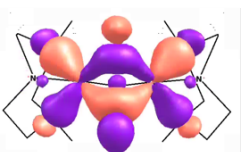

12

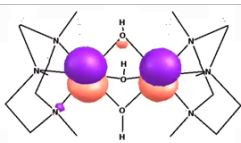

18

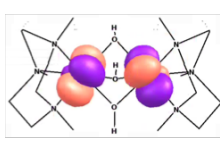

2
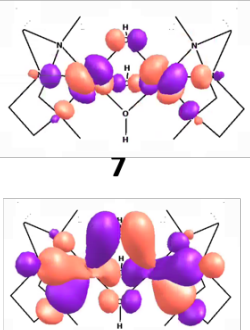

13

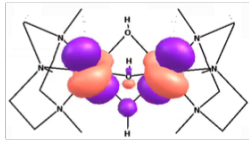

3

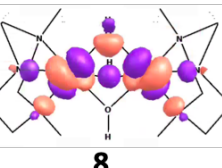

8

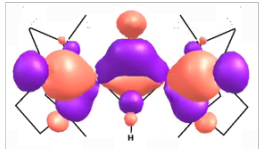

14

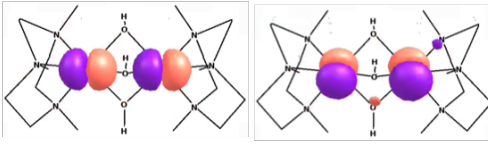

19

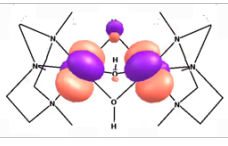

4
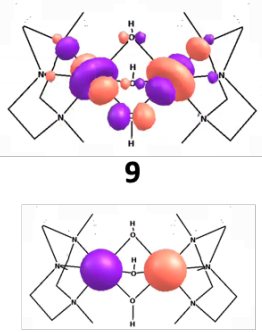

15

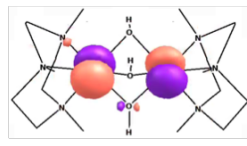

21

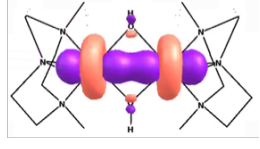

5

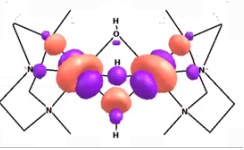

10

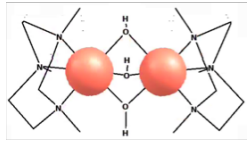

16

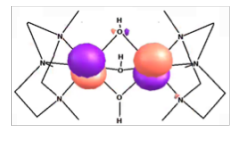

22

Figure S7. Natural molecular orbitals obtained from RASSCF $(30,22), S=2$ calculation.

\section{RASSCF $(30,22), S=3$}

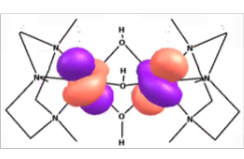

1

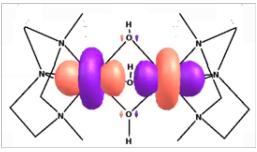

6

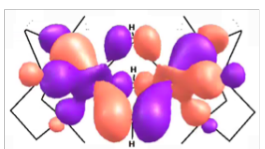

11

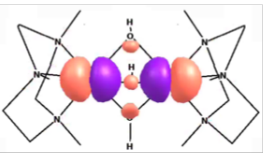

17

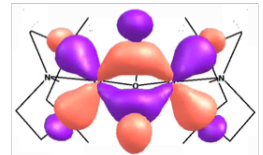

12

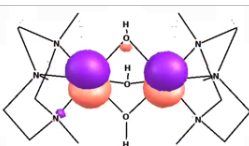

18

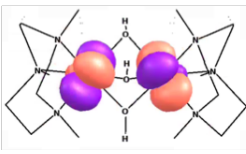

2

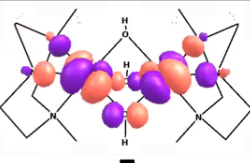

7

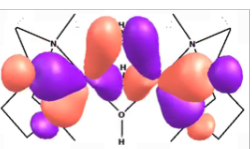

13

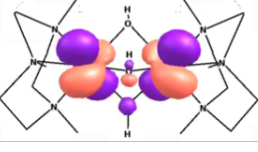

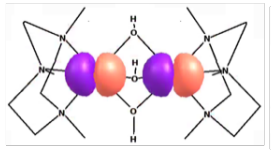

19
3

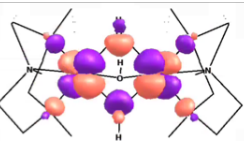

8

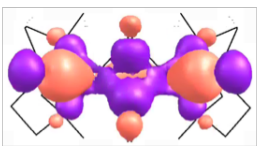

14

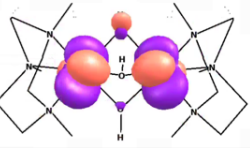

4

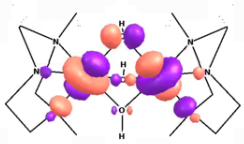

9

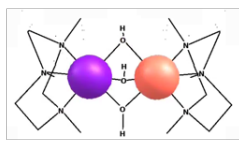

15

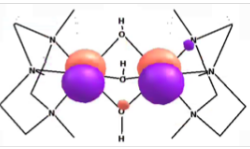

20

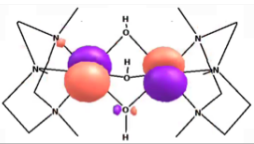

21

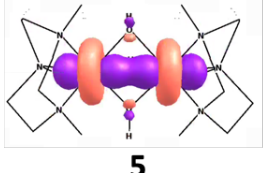

5

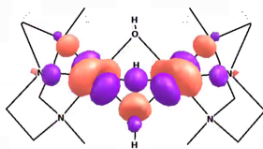

10

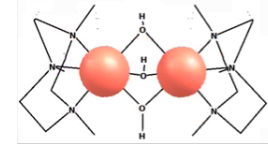

16

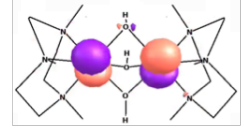

22

Figure S8. Natural molecular orbitals obtained from RASSCF $(30,22), S=3$ calculation. 
Table S1. Occupation numbers of CASSCF $(6,10)$ natural orbitals

\begin{tabular}{|c|c|c|c|c|c|c|c|c|c|c|}
\hline & $\mathbf{1}$ & $\mathbf{2}$ & $\mathbf{3}$ & $\mathbf{4}$ & $\mathbf{5}$ & $\mathbf{6}$ & $\mathbf{7}$ & $\mathbf{8}$ & $\mathbf{9}$ & $\mathbf{1 0}$ \\
\hline Spin 0 & 1.015 & 1.015 & 0.983 & 0.983 & 1.087 & 0.911 & 0.002 & 0.002 & 0.000 & 0.002 \\
\hline Spin 1 & 1.012 & 1.012 & 0.986 & 0.986 & 1.072 & 0.926 & 0.002 & 0.002 & 0.000 & 0.002 \\
\hline Spin 2 & 1.006 & 1.006 & 0.991 & 0.991 & 1.043 & 0.955 & 0.002 & 0.002 & 0.000 & 0.002 \\
\hline Spin 3 & 1.000 & 1.000 & 0.998 & 0.998 & 1.000 & 0.998 & 0.002 & 0.002 & 0.000 & 0.002 \\
\hline
\end{tabular}

Table S2. Occupation numbers of RASSCF $(30,22)$ natural orbitals

\begin{tabular}{|c|c|c|c|c|c|c|c|c|c|c|c|}
\hline \multirow{4}{*}{ Spin 0} & 1 & 2 & 3 & 4 & 5 & 6 & 7 & 8 & 9 & 10 & 11 \\
\hline & 1.020 & 1.020 & 0.986 & 0.986 & 1.109 & 0.898 & 0.013 & 0.014 & 0.013 & 0.014 & 1.996 \\
\hline & 12 & 13 & 14 & 15 & 16 & 17 & 18 & 19 & 20 & 21 & 22 \\
\hline & 1.996 & 1.996 & 1.996 & 1.999 & 1.999 & 1.991 & 1.991 & 1.991 & 1.991 & 1.991 & 1.991 \\
\hline \multirow{4}{*}{ Spin 1} & 1 & 2 & 3 & 4 & 5 & 6 & 7 & 8 & 9 & 10 & 11 \\
\hline & 1.016 & 1.016 & 0.990 & 0.990 & 1.090 & 0.916 & 0.013 & 0.013 & 0.013 & 0.013 & 1.996 \\
\hline & 12 & 13 & 14 & 15 & 16 & 17 & 18 & 19 & 20 & 21 & 22 \\
\hline & 1.996 & 1.996 & 1.996 & 1.999 & 1.999 & 1.991 & 1.991 & 1.991 & 1.991 & 1.991 & 1.991 \\
\hline \multirow{4}{*}{ Spin 2} & 1 & 2 & 3 & 4 & 5 & 6 & 7 & 8 & 9 & 10 & 11 \\
\hline & 1.010 & 1.010 & 0.996 & 0.996 & 1.055 & 0.951 & 0.013 & 0.013 & 0.013 & 0.013 & 1.996 \\
\hline & 12 & 13 & 14 & 15 & 16 & 17 & 18 & 19 & 20 & 21 & 22 \\
\hline & 1.996 & 1.996 & 1.996 & 1.999 & 1.999 & 1.991 & 1.991 & 1.991 & 1.991 & 1.991 & 1.991 \\
\hline \multirow{4}{*}{ Spin 3} & 1 & 2 & 3 & 4 & 5 & 6 & 7 & 8 & 9 & 10 & 11 \\
\hline & 1.003 & 1.003 & 1.003 & 1.003 & 1.003 & 1.003 & 0.013 & 0.013 & 0.013 & 0.013 & 1.996 \\
\hline & 12 & 13 & 14 & 15 & 16 & 17 & 18 & 19 & 20 & 21 & 22 \\
\hline & 1.996 & 1.996 & 1.996 & 1.999 & 1.999 & 1.991 & 1.991 & 1.991 & 1.991 & 1.991 & 1.991 \\
\hline
\end{tabular}


Table S3. Occupation numbers of DMRG-SCF $(30,22)$ natural orbitals

\begin{tabular}{|c|c|c|c|c|c|c|c|c|c|c|c|}
\hline & $\mathbf{1}$ & $\mathbf{2}$ & $\mathbf{3}$ & $\mathbf{4}$ & $\mathbf{5}$ & $\mathbf{6}$ & $\mathbf{7}$ & $\mathbf{8}$ & $\mathbf{9}$ & $\mathbf{1 0}$ & $\mathbf{1 1}$ \\
Spin 0 & 1.021 & 1.021 & 0.986 & 0.986 & 1.110 & 0.896 & 0.015 & 0.015 & 0.015 & 0.016 & 1.994 \\
\cline { 2 - 11 } & $\mathbf{1 2}$ & $\mathbf{1 3}$ & $\mathbf{1 4}$ & $\mathbf{1 5}$ & $\mathbf{1 6}$ & $\mathbf{1 7}$ & $\mathbf{1 8}$ & $\mathbf{1 9}$ & $\mathbf{2 0}$ & $\mathbf{2 1}$ & $\mathbf{2 2}$ \\
& 1.995 & 1.994 & 1.995 & 1.999 & 1.999 & 1.990 & 1.990 & 1.991 & 1.991 & 1.991 & 1.991 \\
\hline \multirow{5}{*}{ Spin 1 } & $\mathbf{1}$ & $\mathbf{2}$ & $\mathbf{3}$ & $\mathbf{4}$ & $\mathbf{5}$ & $\mathbf{6}$ & $\mathbf{7}$ & $\mathbf{8}$ & $\mathbf{9}$ & $\mathbf{1 0}$ & $\mathbf{1 1}$ \\
& 1.017 & 1.017 & 0.989 & 0.989 & 1.091 & 0.916 & 0.015 & 0.015 & 0.015 & 0.015 & 1.994 \\
\cline { 2 - 12 } & $\mathbf{1 2}$ & $\mathbf{1 3}$ & $\mathbf{1 4}$ & $\mathbf{1 5}$ & $\mathbf{1 6}$ & $\mathbf{1 7}$ & $\mathbf{1 8}$ & $\mathbf{1 9}$ & $\mathbf{2 0}$ & $\mathbf{2 1}$ & $\mathbf{2 2}$ \\
& 1.995 & 1.994 & 1.995 & 1.999 & 1.999 & 1.990 & 1.990 & 1.991 & 1.991 & 1.991 & 1.991 \\
\hline \multirow{5}{*}{ Spin 2 } & $\mathbf{1}$ & $\mathbf{2}$ & $\mathbf{3}$ & $\mathbf{4}$ & $\mathbf{5}$ & $\mathbf{6}$ & $\mathbf{7}$ & $\mathbf{8}$ & $\mathbf{9}$ & $\mathbf{1 0}$ & $\mathbf{1 1}$ \\
& 1.011 & 1.011 & 0.996 & 0.996 & 1.056 & 0.950 & 0.016 & 0.016 & 0.016 & 0.016 & 1.994 \\
\hline & $\mathbf{1 2}$ & $\mathbf{1 3}$ & $\mathbf{1 4}$ & $\mathbf{1 5}$ & $\mathbf{1 6}$ & $\mathbf{1 7}$ & $\mathbf{1 8}$ & $\mathbf{1 9}$ & $\mathbf{2 0}$ & $\mathbf{2 1}$ & $\mathbf{2 2}$ \\
& 1.995 & 1.994 & 1.995 & 1.999 & 1.999 & 1.990 & 1.990 & 1.991 & 1.991 & 1.991 & 1.991 \\
\hline \multirow{5}{*}{ Spin 3 } & $\mathbf{1}$ & $\mathbf{2}$ & $\mathbf{3}$ & $\mathbf{4}$ & $\mathbf{5}$ & $\mathbf{6}$ & $\mathbf{7}$ & $\mathbf{8}$ & $\mathbf{9}$ & $\mathbf{1 0}$ & $\mathbf{1 1}$ \\
& 1.003 & 1.003 & 1.003 & 1.003 & 1.003 & 1.003 & 0.016 & 0.016 & 0.016 & 0.016 & 1.993 \\
\cline { 2 - 11 } & $\mathbf{1 2}$ & $\mathbf{1 3}$ & $\mathbf{1 4}$ & $\mathbf{1 5}$ & $\mathbf{1 6}$ & $\mathbf{1 7}$ & $\mathbf{1 8}$ & $\mathbf{1 9}$ & $\mathbf{2 0}$ & $\mathbf{2 1}$ & $\mathbf{2 2}$ \\
& 1.994 & 1.993 & 1.994 & 1.999 & 1.999 & 1.990 & 1.990 & 1.990 & 1.990 & 1.990 & 1.990 \\
\hline
\end{tabular}

CASSCF, $\mathrm{S}=3$

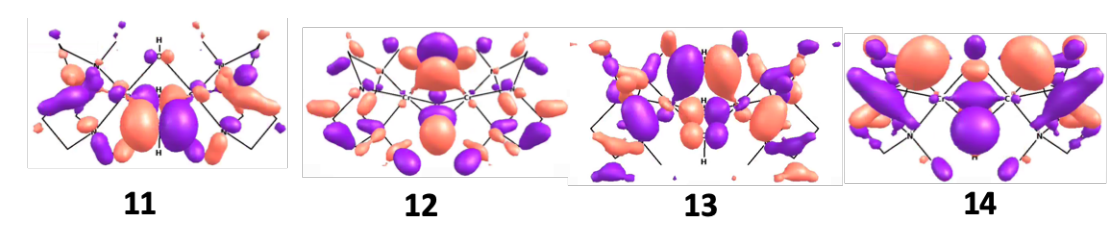

RASSCF, $S=3$

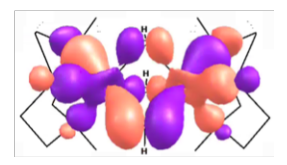

11

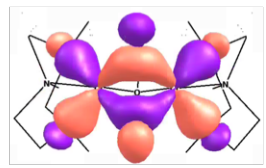

12

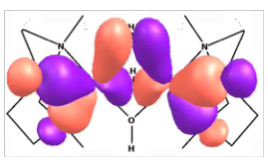

13

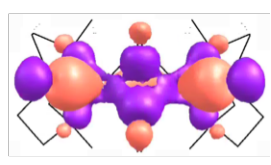

14

Figure S9. Comparison between ligand orbitals in CASSCF vs RASSCF. Figures are plotted at isovalue $=0.04$. 
Table S4. Absolute MCSCF and MC-PDFT energies in hartree atomic units

\begin{tabular}{|c|c|c|c|c|c|}
\hline Method & & Spin 0 & Spin 1 & Spin 2 & Spin 3 \\
\hline & $(\mathrm{e}, \mathrm{o})$ & & & & \\
\hline CASSCF & $(6,10)$ & -3345.601198 & 3345.601010 & -3345.600649 & -3345.600152 \\
\hline MC-PDFT & $(6,10)$ & -3353.451373 & -3353.450978 & -3353.450246 & -3353.449243 \\
\hline RASSCF & $(30,22)$ & -3345.737324 & -3345.737041 & -3345.736499 & -3345.735763 \\
\hline RASPT2 & $(30,22)$ & -3353.415529 & -3353.414872 & -3353.413598 & -3353.412039 \\
\hline & $M$ & & & & \\
\hline DMRG-SCF1 & 500 & -3345.701312 & -3345.700864 & -3345.699083 & -3345.700096 \\
\hline DMRG-PDFT1 & 500 & -3353.437687 & -3353.437304 & -3353.436076 & -3353.434762 \\
\hline DMRG-SCF1 & 1000 & -3345.701592 & -3345.701283 & -3345.700166 & -3345.700249 \\
\hline DMRG-PDFT1 & 1000 & -3353.437545 & -3353.437077 & -3353.435491 & -3353.43466 \\
\hline DMRG-SCF2 & 1000 & -3345.741708 & -3345.741045 & -3345.741408 & -3345.741779 \\
\hline DMRG-PDFT2 & 1000 & -3353.413811 & -3353.413126 & -3353.411694 & -3353.41011 \\
\hline
\end{tabular}


Table S5. Percentage contribution of $\mathrm{Cr}(3 \mathrm{~d})$ and $\mathrm{O}$ atomic orbitals to selected DMRG molecular orbitals

\begin{tabular}{llllll}
\hline Spin $=0$ & & & & & \\
\hline Orbital No. & $\%$ O 2p & \% Cr 3d & Occ. No. & O electrons & Cr electrons \\
\hline 11 & 31.97 & 12.68 & 1.996 & 0.64 & 0.25 \\
12 & 30.68 & 20.07 & 1.996 & 0.61 & 0.40 \\
13 & 31.96 & 13.46 & 1.996 & 0.64 & 0.27 \\
14 & 30.68 & 20.19 & 1.996 & 0.61 & 0.40 \\
1 & 0.24 & 98.64 & 1.020 & 0.00 & 1.01 \\
2 & 0.24 & 98.64 & 1.020 & 0.00 & 1.01 \\
3 & 1.96 & 96.10 & 0.986 & 0.02 & 0.95 \\
4 & 1.96 & 96.07 & 0.986 & 0.02 & 0.95 \\
5 & 2.79 & 93.24 & 1.109 & 0.03 & 1.03 \\
6 & 1.45 & 96.99 & 0.898 & 0.01 & 0.87 \\
7 & 2.00 & 85.62 & 0.013 & 0.00 & 0.01 \\
8 & 4.51 & 82.70 & 0.014 & 0.00 & 0.01 \\
9 & 2.00 & 85.80 & 0.013 & 0.00 & 0.01 \\
10 & 4.51 & 82.76 & 0.014 & 0.00 & 0.01 \\
\hline
\end{tabular}

\begin{tabular}{llllll}
\hline Spin $=3$ & & & & & \\
\hline Orbital No. & $\%$ O 2p & \% Cr 3d & Occ. No. & O electrons & Cr electrons \\
\hline 11 & 31.97 & 12.68 & 1.996 & 0.63 & 0.26 \\
12 & 30.68 & 20.07 & 1.996 & 0.60 & 0.40 \\
13 & 31.96 & 13.46 & 1.996 & 0.63 & 0.26 \\
14 & 30.68 & 20.19 & 1.996 & 0.60 & 0.40 \\
1 & 0.24 & 98.64 & 1.003 & 0.00 & 0.99 \\
2 & 0.24 & 98.64 & 1.003 & 0.00 & 0.99 \\
3 & 1.96 & 96.10 & 1.003 & 0.02 & 0.96 \\
4 & 1.96 & 96.07 & 1.003 & 0.02 & 0.96 \\
5 & 2.79 & 93.24 & 1.003 & 0.03 & 0.93 \\
6 & 1.45 & 96.99 & 1.003 & 0.01 & 0.97
\end{tabular}




\begin{tabular}{llllll}
7 & 2.00 & 85.62 & 0.013 & 0.00 & 0.01 \\
8 & 4.51 & 82.70 & 0.013 & 0.00 & 0.01 \\
9 & 2.00 & 85.80 & 0.013 & 0.00 & 0.01 \\
10 & 4.51 & 82.76 & 0.013 & 0.00 & 0.01 \\
\hline
\end{tabular}

Table S6. Relative energies of spin states and coupling constant $(J)$ in $\mathrm{cm}^{-1}$ for the modified chromium dimer without the bridging hydroxide ligands. This table is based on a separate calculation where the three hydroxide ligands were deleted and the coupling constant was calculated for the modified molecule. We used CAS $(6,10)$ containing six electrons in ten chromium $3 \mathrm{~d}$ orbitals and and RAS $(22,18)$ where 8 orbitals containing Cr $3 \mathrm{~s} / 3 \mathrm{p}$ were in RAS1 while the $3 \mathrm{~d}$ orbitals were in RAS2. Two holes were allowed in RAS1. Both CAS and RAS spaces are analogous to active spaces used for Kremer's dimer.

\begin{tabular}{|c|c|c|c|c|}
\hline \multirow{2}{*}{ Method } & \multicolumn{3}{|c|}{ Energy relative to $S=0$} & \multirow{2}{*}{$J$} \\
\hline & $\mathrm{S}=1$ & $\mathrm{~S}=2$ & $\mathrm{~S}=3$ & \\
\hline CASSCF & 251 & 754 & 1504 & -125 \\
\hline CAS-PDFT & 409 & 1254 & 2761 & -230 \\
\hline RASSCF & 357 & 1074 & 1990 & -166 \\
\hline RAS-PDFT & 402 & 1181 & 2258 & -188 \\
\hline
\end{tabular}


Table S7. Comparison between occupation numbers of Kremer's dimer and dimer with deleted hydroxide ligands.

(a) $\operatorname{Spin}=0$

\begin{tabular}{|c|c|c|c|c|c|c|c|c|c|c|}
\hline & $\mathbf{1}$ & $\mathbf{2}$ & $\mathbf{3}$ & $\mathbf{4}$ & $\mathbf{5}$ & $\mathbf{6}$ & $\mathbf{7}$ & $\mathbf{8}$ & $\mathbf{9}$ & $\mathbf{1 0}$ \\
\hline $\begin{array}{c}\text { Kremer's } \\
\text { dimer }\end{array}$ & 1.02 & 1.02 & 0.98 & 0.98 & 1.09 & 0.91 & 0.00 & 0.00 & 0.00 & 0.00 \\
\hline $\begin{array}{c}\text { Dimer } \\
\text { without } \\
\text { bridge }\end{array}$ & 1.05 & 1.05 & 0.95 & 0.95 & 1.24 & 0.76 & 0.00 & 0.00 & 0.00 & 0.00 \\
\hline
\end{tabular}

(b) $\operatorname{Spin}=1$

\begin{tabular}{|c|c|c|c|c|c|c|c|c|c|c|}
\hline & $\mathbf{1}$ & $\mathbf{2}$ & $\mathbf{3}$ & $\mathbf{4}$ & $\mathbf{5}$ & $\mathbf{6}$ & $\mathbf{7}$ & $\mathbf{8}$ & $\mathbf{9}$ & $\mathbf{1 0}$ \\
\hline $\begin{array}{c}\text { Kremer's } \\
\text { dimer }\end{array}$ & 1.01 & 1.01 & 0.99 & 0.99 & 1.07 & 0.93 & 0.00 & 0.00 & 0.00 & 0.00 \\
\hline $\begin{array}{c}\text { Dimer } \\
\text { without } \\
\text { bridge }\end{array}$ & 1.04 & 1.04 & 0.96 & 0.96 & 1.20 & 0.80 & 0.00 & 0.00 & 0.00 & 0.00 \\
\hline
\end{tabular}

(c) $\operatorname{Spin}=2$

\begin{tabular}{|c|c|c|c|c|c|c|c|c|c|c|}
\hline & $\mathbf{1}$ & $\mathbf{2}$ & $\mathbf{3}$ & $\mathbf{4}$ & $\mathbf{5}$ & $\mathbf{6}$ & $\mathbf{7}$ & $\mathbf{8}$ & $\mathbf{9}$ & $\mathbf{1 0}$ \\
\hline $\begin{array}{c}\text { Kremer's } \\
\text { dimer }\end{array}$ & 1.01 & 1.01 & 0.99 & 0.99 & 1.04 & 0.96 & 0.00 & 0.00 & 0.00 & 0.00 \\
\hline $\begin{array}{c}\text { Dimer } \\
\text { without } \\
\text { bridge }\end{array}$ & 1.02 & 1.02 & 0.98 & 0.98 & 1.13 & 0.87 & 0.00 & 0.00 & 0.00 & 0.00 \\
\hline
\end{tabular}

(d) $\operatorname{Spin}=3$

\begin{tabular}{|c|c|c|c|c|c|c|c|c|c|c|}
\hline & $\mathbf{1}$ & $\mathbf{2}$ & $\mathbf{3}$ & $\mathbf{4}$ & $\mathbf{5}$ & $\mathbf{6}$ & $\mathbf{7}$ & $\mathbf{8}$ & $\mathbf{9}$ & $\mathbf{1 0}$ \\
\hline $\begin{array}{c}\text { Kremer's } \\
\text { dimer }\end{array}$ & 1.01 & 1.01 & 0.99 & 0.99 & 1.04 & 0.96 & 0.00 & 0.00 & 0.00 & 0.00 \\
\hline $\begin{array}{c}\text { Dimer } \\
\text { without } \\
\text { bridge }\end{array}$ & 1.02 & 1.02 & 0.98 & 0.98 & 1.13 & 0.87 & 0.00 & 0.00 & 0.00 & 0.00 \\
\hline
\end{tabular}


(a)

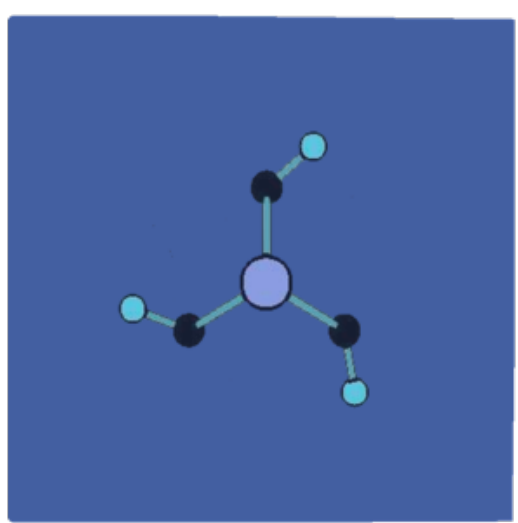

CAS-PDFT (S=0)

(b)

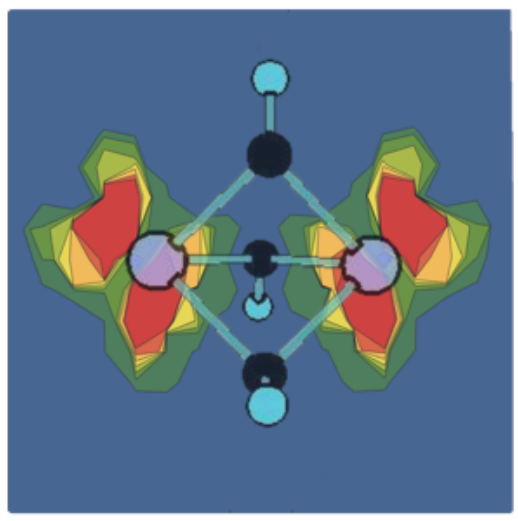

CAS-PDFT $(\mathbf{S}=\mathbf{0})$

(c)

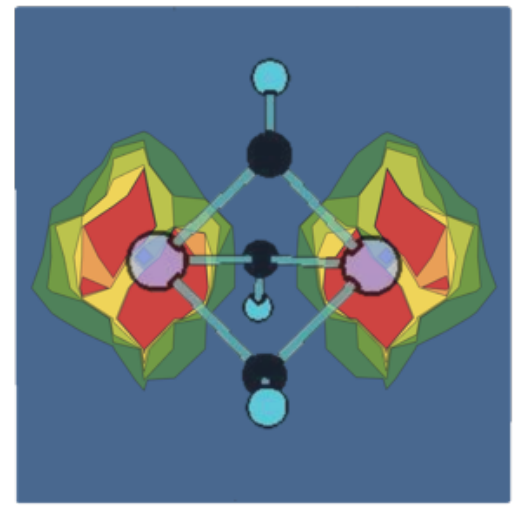

CAS-PDFT $(\mathbf{S}=\mathbf{0})$
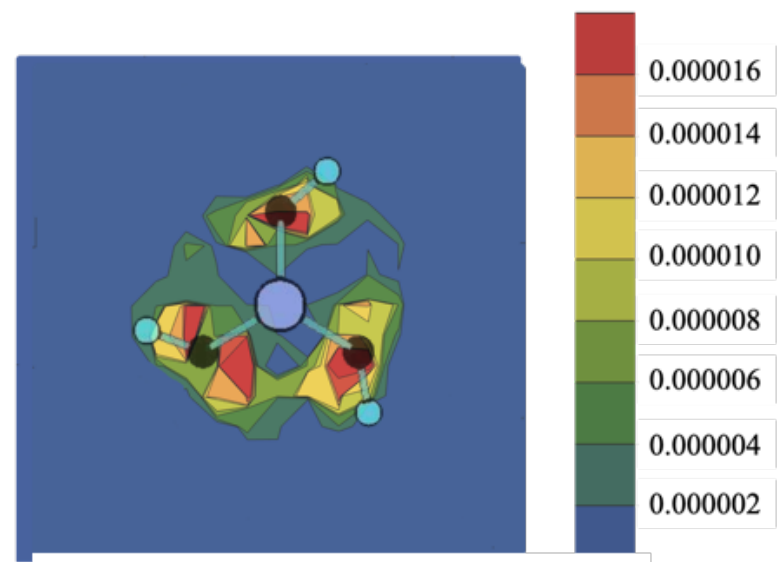

\section{DMRG1-PDFT (S=0)}
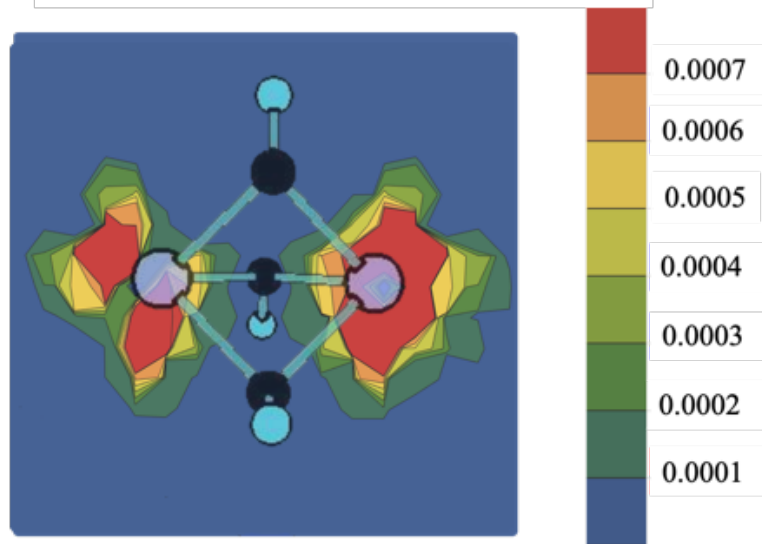

DMRG1-PDFT $(\mathbf{S}=\mathbf{0})$
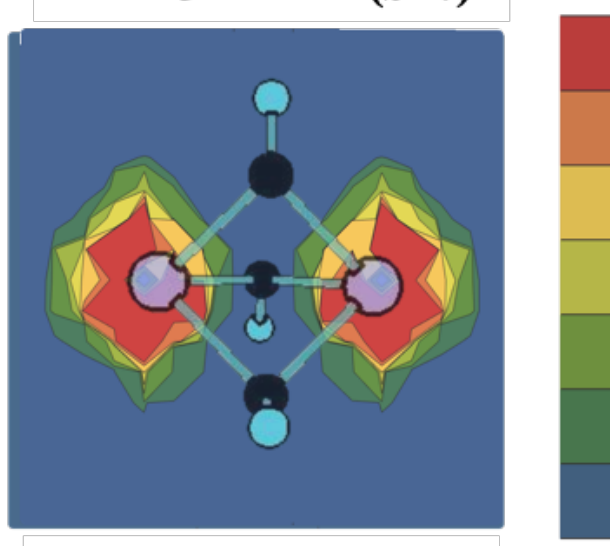

DMRG1-PDFT (S=0)

Figure S10. Unpaired density for CAS-PDFT (left) and DMRG1-PDFT (right) for Kremer's complex in the (a) YZ-plane, (b) XZ-plane, and (c) XY-plane. The colors purple, black and blue corresponds to chromium, oxygen and hydrogen atoms, respectively. 
(a)

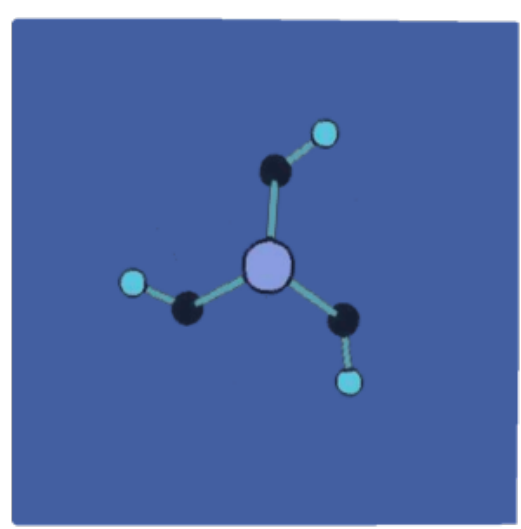

CAS-PDFT (S=0)

(b)

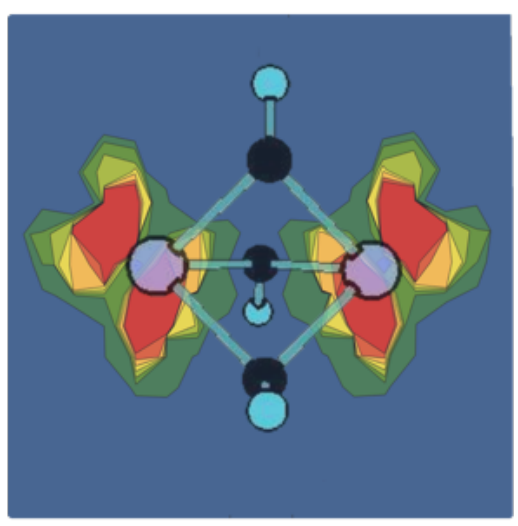

CAS-PDFT ( $\mathbf{S}=\mathbf{0})$

(c)

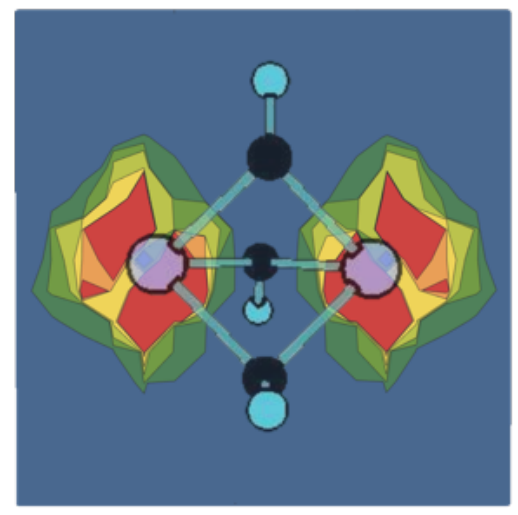

CAS-PDFT $(\mathbf{S}=\mathbf{0})$
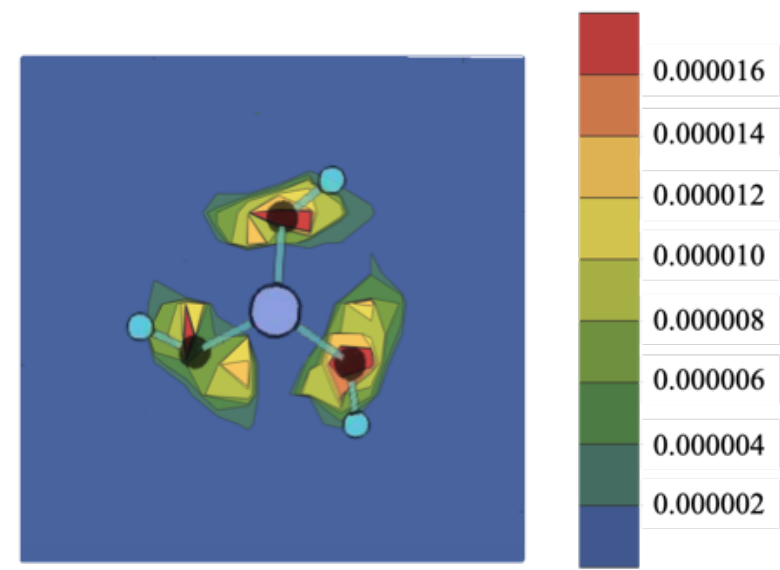

RAS-PDFT (S=0)
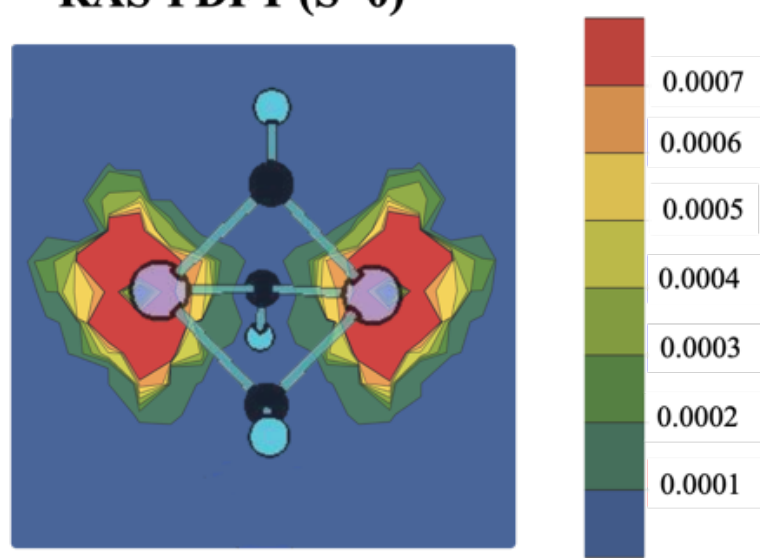

RAS-PDFT (S=0)
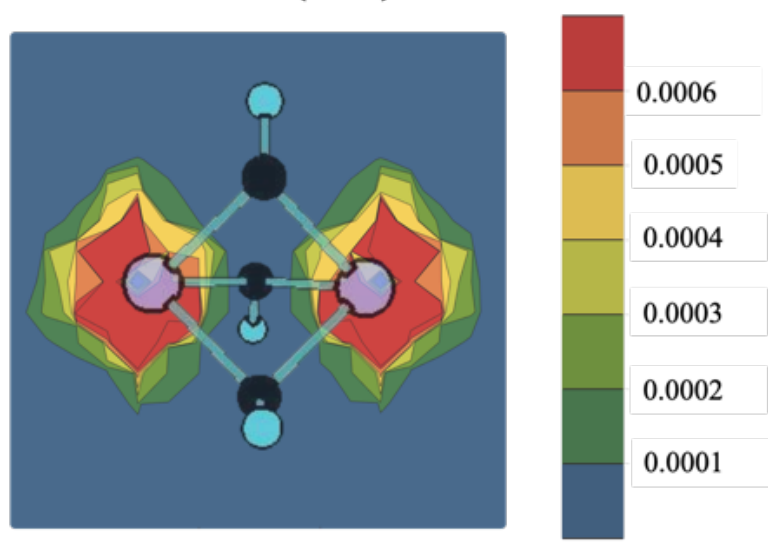

RAS-PDFT (S=0)

Figure S11. Unpaired density for CAS-PDFT (left) and RAS-PDFT(right) for Kremer's complex in the (a) YZ-plane, (b) XZ-plane, and (c) XY-plane. The colors purple, black and blue corresponds to chromium, oxygen and hydrogen atoms, respectively. 

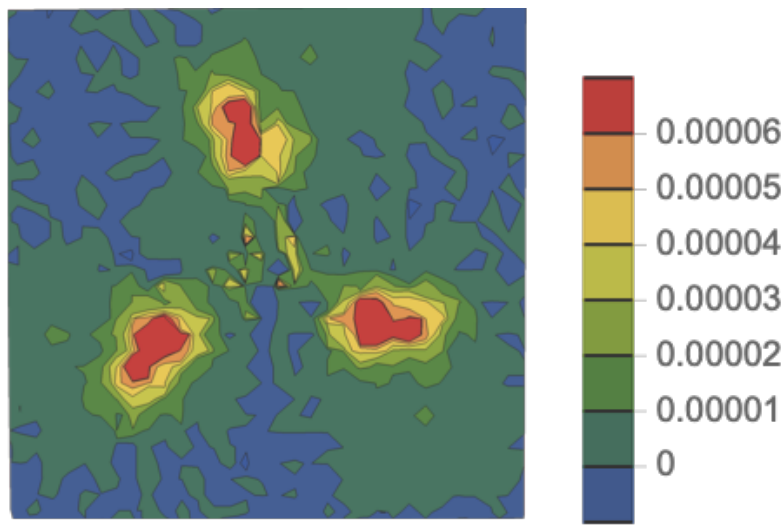

YZ Plane
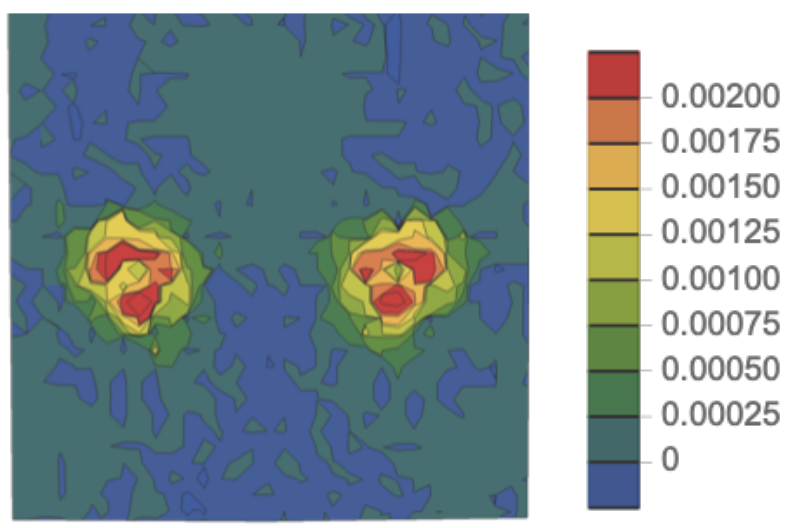

XZ Plane

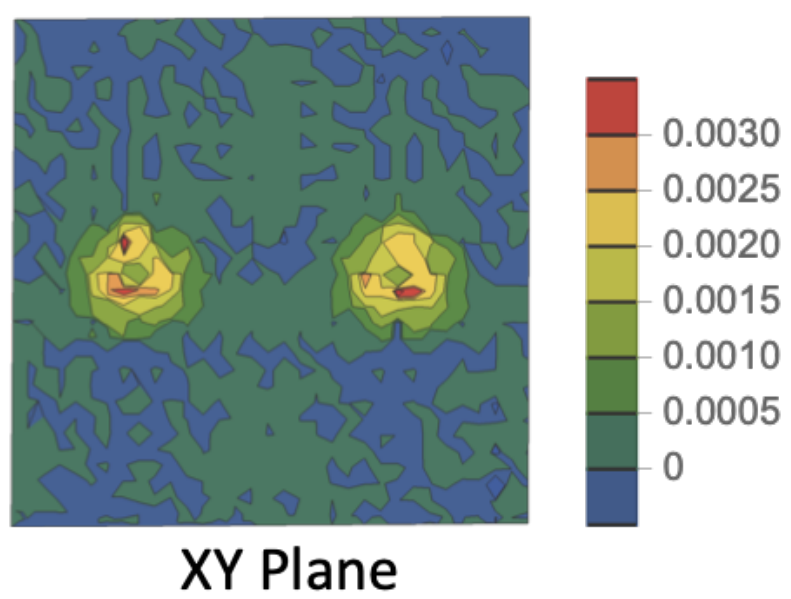

Figure S12. Unpair electron density difference between $S=3$ (RAS-PDFT) and $S=0$ (RASPDFT). The positive values (green-red) shows $S=3$ has larger unpair density in that region while blue color depicts higher unapair density in $\mathrm{S}=0$ state. 